\title{
REVIEW OF SMART SENSOR NETWORKS FOR ENVIRONMENT MONITORING
}

\author{
Satvir Singh ${ }^{1}$, Rajeshwar Singh ${ }^{2}$ \\ ${ }^{1}$ Assistant Professor, Electronics and Communication Engineering, Chandigarh Engineering College, India \\ ${ }^{2}$ Director, DOABA Group of Colleges, Campus -III, Rahon, Nawanshar, Punjab
}

\begin{abstract}
This review article focuses on the various environment monitoring systems developed over the years. The development of any monitoring system is dependent on certain key factors namely cost of the system, energy efficiency of the system, signal to noise ratio, interference rejection during varying atmospheric conditions. The complexity of any system depends upon the number of nodes the system requires to monitor, the more the number of nodes the more complex structure becomes. If we use smart sensor network with each node independently functioning and transmitting data to one parent node that performs required processing the complexity decreases.
\end{abstract}

Keywords: Zig bee, raspberry pi, smart sensor network, wireless communication, embedded controller, ARM, Wi-fi

\section{INTRODUCTION}

Since India is still a developing country, major part of the energy production is based on natural resources like coal, natural gas etc., the energy produced from these resources is not clean energy and leaves a highly toxic trail behind it. The result of which is the pollutant concentration in atmosphere has exceeded levels sustainable for good health conditions. Today the emerging need is a monitoring system that senses all the pollutants from the surroundings and sends the data to control center to take necessary action. According to United Nations Environmental Program the buildings consume a humongous $40 \%$ of the all the energy casing $35 \%$ of total pollution, if we carefully monitor such areas we are hopeful that we can cut down major pollutants from air. The major contributors of the greenhouse emissions are carbon dioxide, oxides of sulphur and nitrogen, suspended particulate matter and aerosols.

An uncontrolled and monitored system leads to health hazards to the population living within or around that area. So in order to improve the health conditions of the population it is very crucial that we monitor and control such pollutants.

Environment monitoring systems till now have been implemented using centrally controlled system based on various micro-controller technologies available.in such a system the entire work load is on the central unit whereas the nodes or the sensors do the work of sensing the stimuli only. In case there is a malfunctioning of the central system the entire monitoring system goes down, also if one of the sensors malfunctions than till the time it is not restored the system is affected whereas with a smart sensor network the nearest similar node is switched on to prevent the malfunctioning of the system, removing the latency that may come in such cases.
Any environment monitoring system comprises of three main modules which are Standard Transducer Interface Module (STIM), Graphical User Interface and Transducer Independent Interface. The STIM is used for gathering the data, GUI is used for displaying the relevant data in perceivable form and T.II is used to communicate this data to the Network Capable Application Processor

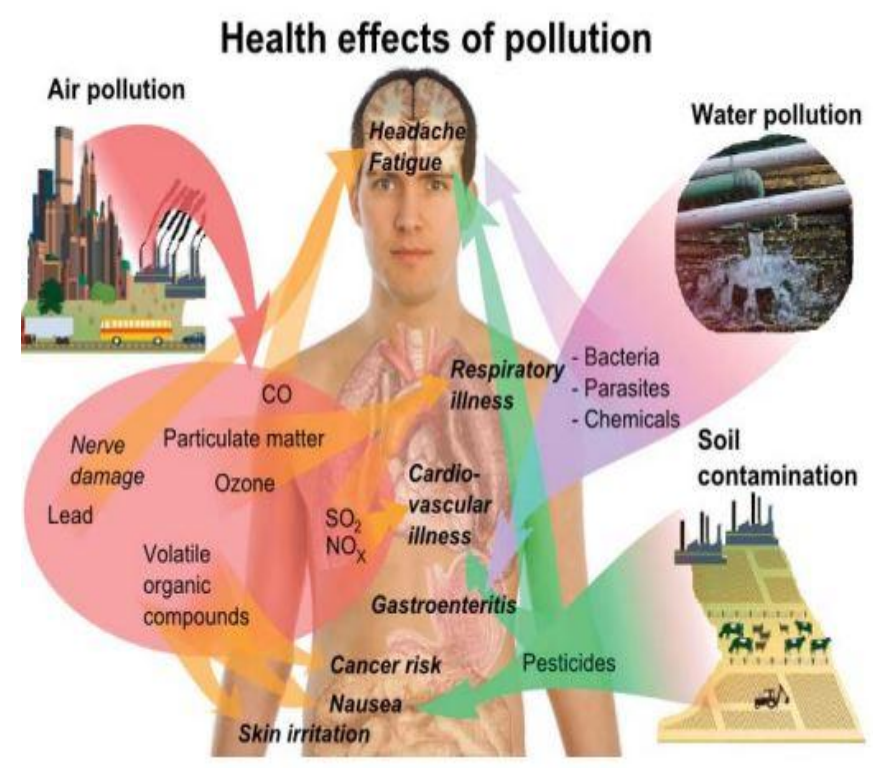

Fig 1: Health Hazards posed by various Pollutants 


\section{METHODOLOGY}

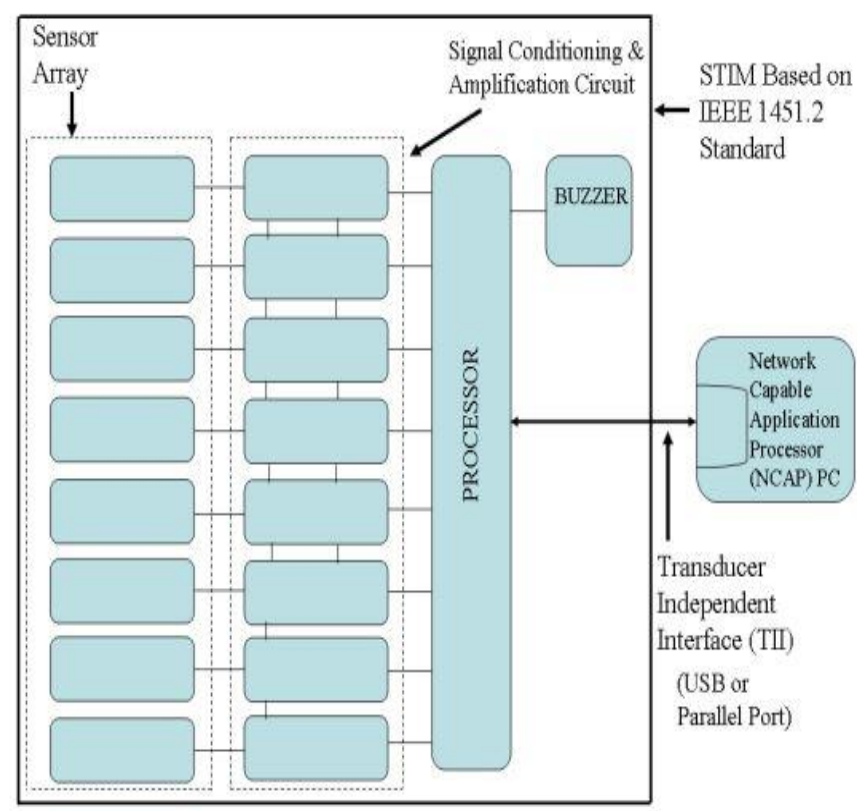

Fig 2: General Block of an Environment Monitoring System

By having a precise control of the environment one can increase the standard of living of all individuals as many of the health problems are created by these pollutants. Controlling may also incorporate remote controlled devices which are a boon for disabled and aged population of the country but the problem is that the existing systems are very costly and difficult to maintain as a result they are not thought of as cost effective method of monitoring.

This paper takes into account the foundation of all such systems and existing systems built on these foundations.

\section{STANDARD TRANSDUCER INTERFACE MODEL (STIM)}

STIM is based on the IEEE 1451 standard which consists of a collection of Transducer Electronic Data Sheets, transducer independent interface and connection protocol between the network and system. It is desired that a transducer should be such that it not only senses the stimulus but also conditions the signal so that the control system can easily interpret reducing the work load on the system.

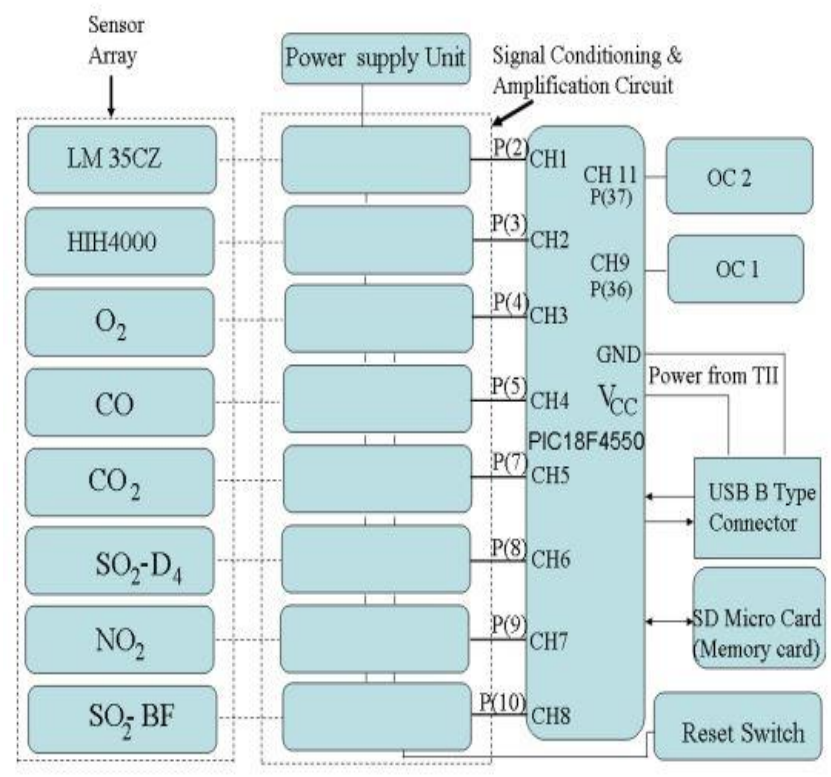

Fig 3: An STIM Model

\subsection{Sensor Array}

Sensor is any device that senses a particular stimulus only and provides an output in the form of an electrical signal.

A) Gas Sensor: This category of the sensors is most difficult to fabricate and use in any system as the output of such sensor is in the form of analog signal that varies depending upon the concentration the gas in the area. A very precise translation of the analog signal to electrical domain is required to successfully implement the sensor. Some of the sensors that are used are based on chemical sensing, photo ionic sensing but the most successful are the MEMS based sensors.

B) Temperature and Humidity Sensor: Recent advances in these sensors have enabled us to integrate the two on a single chip and also a signal conditioner on the same chip as a result we get a digital output from the SOC. The sensor is based on a linear temperature coefficient of a metal for temperature sensing and chemical ionic sensor for the humidity sensing.

C) Light Sensor: Use of artificial lights bulbs, CFL etc. is very important in any area but these are over used at all times. A light sensor carefully monitors the intensity of the light that is coming and provides a digital output so that the lights can be increased, decreased or turned off depending on output signal. This sensor is based on photon detection principle.

For a very large area a network of such sensors can be used each communicating with the central command center

\section{TRANSDUCER INDEPENDENT INTERFACE}

TII takes into account the protocols or methods used for communication of sensed data to the control center. Many systems have been developed for the same depending upon 
area to be covered and cost constraints, they may be both wireless and wired.

A) Wired: In a wired system the sensors are connected to the control center using wires the major disadvantage of such system is the losses incurred in wired transmission due to resistance of wire and the cost of laying the wire. As a result over the years this has become least preferred method of developing a system.

B) Wireless: In a wireless system a number of communication technologies can be used like radio frequency, Bluetooth, infrared etc. All these technologies have a built in error control module as a result of which we get a far more reliable system without the complication of the wired technology.

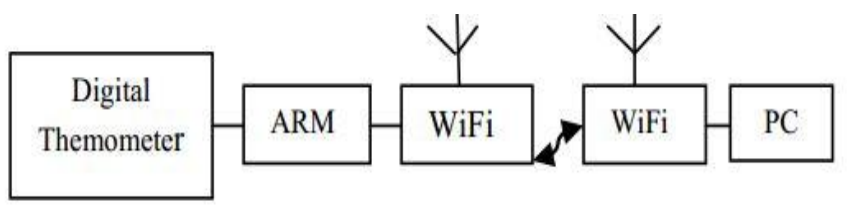

Fig 4: General Block of Wireless Network

\section{NETWORK CAPABLE APPLICATION PROCESSOR}

NCAP is mode of representation of data in user readable form. It consists of two components namely; support and application part.

The support block consists of the network interface, transducer interface and an operating system. The application block consists of a Processor that gives an appropriate output in form of display and may also control the devices to take necessary actions based on the input received.

NCAP may be in form of a computer, tablet, mobile device etc.

\section{MARKET AVAILABLE SYSTEMS}

Honey Well is the largest vendor of environment monitoring and control systems. The cost of such systems is very high and the complexity at which these systems work is great as a result they are not much user friendly. They also require timely service and maintenance as a result the overall cost becomes totally unacceptable for a common man.

The technologies available are largely based on microcontroller thereby limiting its network abilities in terms of number of nodes that can be successfully implemented as multiplexing a sensor pin would lead to latency of system thus every sensor occupies one input pin and for a very large area multiple controllers may be required finally increasing the cost and maintenance.

Recently developed technology is based in using zigbee modules, but not much research has been done in this field largely because of the fact that it's comparatively new.
Using a zig bee network largely simplifies the entire operation as it has its own independent TII and can be easily interfaced with a microprocessor running a Linux operating system and later displaying the result on the internet making the system accessible from anywhere around the world increasing the ease of use.

\section{CONCLUSION}

Although the requirement of an environment monitoring system is very important but still due to higher cost and complexity it has been inaccessible for normal use. If technologies like zigbee are further simplified than a modular system can be achieved with a very low cost.

\section{REFERENCES}

[1]. "UNEP sustainable buildings and climate initiative (SBCI)," United Nations Environmental Programme report, Tech. Rep., 2009.

[2]. "AWO Asia and world outlook," Institute of Energy Economics, Tokyo, Japan, Tech. Rep., 2007.

[3]. R. K. Pachauri and A. Reisinger, "Climate change 2007impacts, adaptation and vulnerability," Contribution of Working Group II to the $4^{\text {th }}$ Assessment Report of the IPCC, IGeneva, Switzerland, Rep. 1352, 2007.

[4]. "IEO international energy outlook," Energy Information Administration, Washington, DC, Tech. Rep., 2009.

[5]. "Indoor environment monitoring," ASHRAE Hand Book, Hong Kong: ASHRAE, 2001, ch. 9, pp. 9.1-9.20.

[6]. C. K. Chau, W. K. Hui, and M. S. Tse, "Evaluation of health benefits for improving indoor air quality in work place," Environ. Int., vol. 33, no. 2, pp. 186-198, 2007.

[7]. W. S. Cain, J. M. Samet, and M. J. Hodgson, "The quest for negligible health risks from indoor air," ASHRAE J., vol. 37, no. 7, p. 38, 1995.

[8]. G. Hoek, B. Brunekreef, S. Goldbohm, P. Fischer, and P. A. Brandt, "Association between mortality and indicators of traffic-related air pollution in the Netherlands: A cohort study," Lancet vol. 360, no. 9341, pp. 1184-1185, Oct. 2002.

[9]. J. F. Nicol and M. A. Humphreys, "Adaptive thermal comfort and sustainable thermal standards for buildings," Energy Build., vol. 34, no. 6, pp. 563-572, 2002.

[10]. G. S. Brager and R. J. Dear, "Thermal adaptation in the built environment: A literature review," Energy Build., vol. 27, no. 1, pp. 83-96, 1998.

[11]. M. Fountain, G. Brager, and R. J. Dear, "Expectations of indoor climate control," Energy Build., vol. 24, no. 3, pp. 179-182, 1996.

[11]. M. Fountain, G. Brager, and R. J. Dear, "Expectations of indoor climate control," Energy Build., vol. 24, no. 3, pp. 179-182, 1996.

[12]. Thermal Environmental Conditions for Human Occupancy, American Society of Heating, Refrigerating and Air-conditioning Engineers Inc., ASHRAE 55, 2004.

[13]. A. Kumar, I. P. Singh, and S. K. Sud, "Development of multi-channel data logger for indoor environment," J. Eng., vol. 2, no. 9, pp. 690-697, 2010. 
[14]. C. Arnold, M. Harms, and J. Goschnick, "Air quality monitoring and fire detection with the Karlsruhe electronic micronose KAMINA," IEEE Sensors J., vol. 2, no. 3, pp. 179-188, Jun. 2002.

[15]. N. Kularatna and B. H. Sudantha, "An environmental air pollution monitoring system based on the IEEE 1451 standard for low cost requirements," IEEE Sensors J., vol. 8, no. 4, pp. 415-422, Apr. 2008.

[16]. F. Sarry and M. Lumbreras, "Gas discrimination in airconditioned system," IEEE Trans. Instrum. Meas., vol. 49, no. 4, pp. 809-812, Apr.2000.

[17]. H. M. G. Ramos, J. M. D. Pereria, V. Viegas, O. Postolache, and P. M. B. S. Girao, "A virtual instrument to test smart transducer interface modules (STIMS)," IEEE Trans. Instrum. Meas., vol. 53, no. 4, pp. 1232-1239, Apr. 2004.

[18]. D. Grimaldi and M. Marinov, "Distrebuted measurement system," Measurement, vol. 30, no. 4, pp. 279-287, 2001.

[19]. D. D. Lee and D. S. Lee, "Environment gas sensors," IEEE Sensors J., vol. 1, no. 3, pp. 214-215, Oct. 2001.

[20]. P. O. Fanger, "Thermal environment - human requirements," Environmentalist, vol. 6, no. 4, pp. 275-278, 1986.

[21]. Y. Kim, R. G. Evans, and W. M. Iversen, "Remote sensing and control of an irrigation system using a distributed wireless sensor network," IEEE Trans. Instrum. Meas., vol. 57, no. 7, pp. 1379-1387, Jul. 2008.

[22]. H. C. Lee, A. Banerjee, Y. M. Fang, B. J. Lee, and C. T. King, "Design of a multifunctional wireless sensor network for in situ monitoring of debris flows," IEEE Trans. Instrum. Meas., vol. 59, no. 11, pp. 2958-2967, Nov. 2010. [23]. A. Carullo, S. Corbellini, M. Parvis, and A. Vallan, "A wireless sensor network for cold chain monitoring," IEEE Trans. Instrum. Meas., vol. 58, no. 5, pp. 1405-1411, May 2009.

[24]. A. Lay-Ekuakille and A. Trotta, "Predicting VOC concentration measurements: Cognitive approach for sensor networks," IEEE Sensors J., vol. 5, no. 11, pp. 3923-3030, Nov. 2011.

[25]. A. Lay-Ekuakille, P. Vergallo, N. I. Giannoccaro, A. Massaro, and D. Caratelli, "Prediction and validation of outcomes from air monitoring sensors and networks of sensors," in Proc. 5th Int. Conf. Sensing Technol., Nov.Dec. 2011, pp. 73-78.

[26]. IEEE Standard for a Smart Transducer Interface for Sensors and Actuators - Transducer to Microprocessor Communication Protocols and Transducer Electronic Data Sheet (TEDS) Formats, IEEE standard 1451.2, 1997.

[27]. IEEE Standard for a Smart Transducer Interface for Sensors and Actuators-Network Capable Application Processor (NCAP) Information Model, IEEE standard 1451.1, 1999.

[28]. A. Kumar, I. P. Singh, and S. K. Sud, "Energy efficient and low cost indoor environment monitoring system based on the IEEE 1451 standard," IEEE Sensors J., vol. 11, no. 10, pp. 2598-2610, Oct. 2011.

[29]. M. A. Abas, M. H. Fadzil, and A. K. Hakiim, "Development of environmental monitoring data management system using OSS python," World Academy Sci., Eng. Technol., vol. 64, pp. 180-185, Sep. 2010.
[30]. J. B. Miller, "Catalytic sensors for monitoring explosive atmospheres," IEEE Sensors J., vol. 1, no. 1, pp. 88-93, Jun. 2001.

[31]. "Solid state gas sensors," in International Sensor Technology. Mountain View, CA: IST, ch. 4, pp. 47-52, 2009.

[32]. "Infrared gas sensors," in International Sensor Technology. Mountain View, CA: IST, ch. 5, pp. 55-71, 2008.

[33]. "Photoionization detectors," in International Sensor Technology. Mountain View, CA: IST, ch. 6, pp. 73-81, 2009.

[34]. D. M. Wilson, S. Hoyt, J. Janata, K. Booksh, and L. Obando, "Chemical sensors for portable, hand held field instruments," IEEE Sensors J., vol. 1, no. 4, pp. 256-274, Dec. 2001.

\section{BIOGRAPHIES}

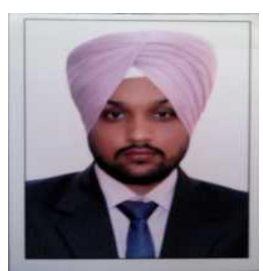

Satvir Singh is currently doing his M. Tech from Doaba Institute of Engineering and technology, Kharar, Mohali. He did his B.E. from Chandigarh College of Engineering and Technology, Chandigarh. He has one and half year of Research Experience and 4 years of teaching experience.

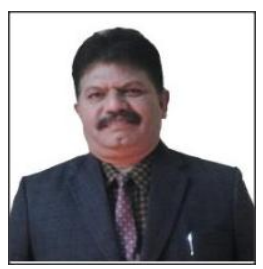

Dr. Rajeshwar Singh is Director at, DOABA Group of Colleges, Campus III, Rahon, Nawanshar, Punjab. He is $\mathrm{Ph}$. D. from Vinoba Bhave University 